\title{
Complete Interruption of Aortic Arch and Non-Immune Hydrops Fetalis: A Case Report with Autopsy
}

\author{
Cesar Cilento Ponce
}

Universidade Metropolitana de Santos (UNIMES), Santos, São Paulo, SP - Brazil

\section{Introduction}

Interrupted aortic arch (IAA) occurs in three births per million, and accounts for approximately $1 \%$ of congenital heart diseases (CHDs). ${ }^{1-3}$ The first case reported with these anomalies appears to be the one by Seidel in $1818 .^{1}$

IAA is a severe form of CHD characterized by a lack of luminal continuity between the ascending and the descending thoracic aorta. ${ }^{4}$ The two most common associated cardiac anomalies are ventricular septal defect $(90 \%)$ and patent ductus arteriosus (98\%). ${ }^{4}$ Isolated IAA is very uncommon ${ }^{2,4}$ and it is considered incompatible with life. ${ }^{3}$

To understand the pathogenesis of IAA, we must remember the embryological development of the main vessels. At approximately the third week of embryonic life, two aortas, a dorsal and a ventral, are connected by six paired arterial branches (aortic arches). In the course of development, a series of changes occur leading to the disappearance of several aortic arches, beginning with the first, the second and the fifth pairs. The third pair of arches persists to form the common carotid and external carotid arteries, and the fourth arches persist to form the permanent arch of the aorta on the left side and the proximal segment of the subclavian artery on the right side. The proximal portions of the sixth arches become the pulmonary arteries. On the right side, the distal segment disappears, while on the left the corresponding distal segment remains as the ductus

\section{Keywords}

Vascular Ring; Aorta/abnormalities; Aorta/surgery; Aortic Coarctation; Ductus Arteriosus; Perinatal Mortality; Autopsy. arteriosus. ${ }^{1}$ In IAA, there is a failure of development of the fourth left aortic arch, the pulmonary artery that transports the blood from the heart to the descending aorta via the ductus arteriosus. This absence of the aortic isthmus causes a discontinuity between the ascending and descending aorta. ${ }^{5}$

Symptoms usually occur early in the neonatal period and clinical deterioration is often rapid. The median age at death in untreated IAA with associated cardiac anomalies is 10 days. $^{2}$ In the embryo, the superior vena cava returns venous blood from the upper body. This less oxygenated blood leaves the heart via the pulmonary artery. A small amount reaches the lungs while the rest goes into the descending aorta through the ductus arteriosus. The umbilical vein transports oxygenated blood to the right auricle via the hepatic veins, ductus venosus and inferior vena cava; the main part flows through the ventricular septal defect into the left side of the heart, ${ }^{2}$ and from there it is distributed to the head and arms. ${ }^{1,2}$ In this type of circulation, in case of complete loss of continuity between the ascending and descending aorta it is important the re-establishment of a communication that allows the oxygenated blood that reaches the left heart passes into the right side for adequate supply of the lower portion of body. ${ }^{2}$ The absence of a septal defect and a patent ductus arteriosus is incompatible with extra-uterine life.

Prenatal diagnosis of IAA has been reported in few case series. ${ }^{6}$ The prenatal characterization of different IAA types based on echocardiographic examination has some limitations. ${ }^{6}$ The clinician's knowledge of embryology and anatomy of the great vessels is essential for the diagnosis of IAA.

The aim of this study was to draw attention for possible cardiac abnormalities in hydropsy fetus and to report a case of fetal death by IAA with autopsy. 


\section{Case report}

A multiparous 43-year-old woman in prenatal care with negative serological tests for infectious diseases was referred for fetal cardiovascular evaluation at 33 weeks of gestation. Her past medical and family histories were unremarkable. A morphology ultrasound in the third trimester showed a hydrops fetus with probable atrioventricular septal defect (AVSD) and heart failure with pericardial effusion in utero. Fetal echocardiography was not performed. Based on perinatal outcomes, amniocentesis for fetal karyotyping was recommended, but the parents declined. Examination during the 35 weeks' follow-up visit revealed that the fetus had died. A female stillborn infant was delivered vaginally. Permission for autopsy was obtained from the parents.

\section{Autopsy findings}

An autopsy was performed based on the guidelines of the Committee of the College of American Pathologists. ${ }^{7}$ The stillborn weighed 1,510 grams and measured 40 $\mathrm{cm}$ in length. The pertinent findings were as follows: macerated skin, hydrops fetalis facies and no other external malformation. At this time, the umbilical cord blood was already clotted, not allowing a suitable sample for the karyotype.

The cephalic, thoracic, and abdominal organs showed moderate autolysis. The heart was dilated and had increased weight for the gestational age, with $20.5 \mathrm{~g}$ (mean reference value [mRV]; $14.5 \pm 3.7 \mathrm{~g}$ ) and discrete hydropericardium. The ascending aorta ended at the innominate and left common carotid arteries (Figure 1A). The descending thoracic aorta was a continuation of the pulmonary artery with a widely dilated ductus arteriosus (Figure 1B). The left subclavian artery originated from the descending aorta (Figure 1B). There was a complete absence of the segment of aorta between the origin of the left common carotid and the left subclavian arteries (absence of the aortic isthmus). The venous return to the heart was normal. The aortic and pulmonary valves showed no abnormalities (Figure 2A and $2 \mathrm{~B})$. Proximally to the pulmonary artery opening, we noticed the ostium of the left pulmonary artery and distally the ostium of the right pulmonary artery (Figure 2A), and a single coronary artery ostium was identified (Figure 2B). There was a complete AVSD, Rastelli's type $\mathrm{A},{ }^{8}$ with both atrial and ventricular septal defect and common atrioventricular valve that bridges both sides of heart (Figure 2B). The lungs were immature. The liver was enlarged, weighing $111.5 \mathrm{~g}$ (mean reference value $[\mathrm{mRV}] ; 81.8 \pm 22.3 \mathrm{~g}$ ). No other syndromic features or other malformations were noted.

The placenta weighed 220 grams previously fixed in formaldehyde was received in the Pathology Laboratory, which precluded a karyotype test. Histological sections stained with hematoxylin and eosin (H\&E) revealed acute purulent inflammation of the fetal membranes and large areas of placental infarction. No changes were detected in the umbilical cord.

\section{Discussion}

IAA was anatomically classified by Celoria and Patton in $1959^{\circ}$ according to the level of arch interruption: type A, IAA distal to the left subclavian artery, accounting for approximately $30 \%-40 \%$ of cases; type B, IAA between the left common carotid and left subclavian arteries; it is the most common form, representing 53\% of cases; and type $C$ the arch is interrupted between the innominate and left common carotid arteries. This is the most uncommon, accounting for about $4 \%$ of cases. ${ }^{2,4}$ The case reported herein can be classified as type B based on the site of aortic arch interruption. This subtype is found when the left fourth arch segment regresses early, prior to cephalad migration of the left subclavian artery. ${ }^{4}$

IAA type B is usually syndromic; it is the most common cardiac defect occurring in DiGeorge syndrome, which is associated with microdeletions of the segment 22q11.2.3.6 Although fetal karyotyping was indicated by the medical team, the parents did not agree with it.

In our case, the blood from the vena cava emptied into the right atrium and then into the right ventricle. Because of complete AVSD, part of this blood passed into the left atrium and into the left ventricle. From the pulmonary artery, some of this blood flew into the branches of this vessel, and the other part emptied through the ductus arteriosus into the descending aorta. ${ }^{1,4,5}$ In intrauterine life, the fetus receives the oxygenated blood from the placenta through the umbilical vein. The intercavitary flow promoted by septal defect and ductus arteriosus allows a mixed-blood condition sine qua non for survival. ${ }^{6}$ However, in the case described here, the septal defect and the large ductus arteriosus exerted no positive effect on gestational outcome.

Chorioamnionitis is an inflammation of the fetal membranes due to infection. The presence of polymorphonuclear leukocytes indicates acute 

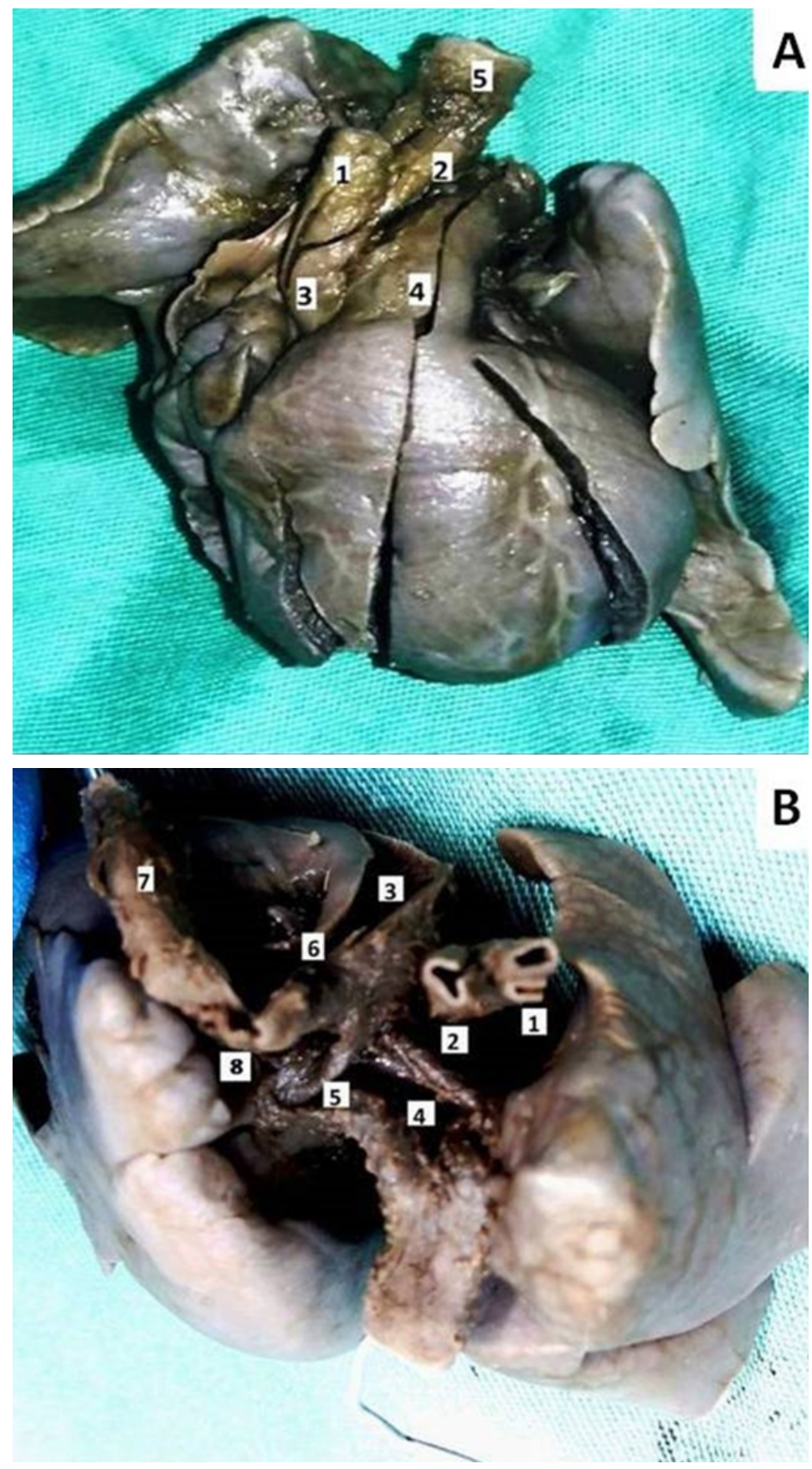

Figure 1 - A: Ascending aorta ending in innominate and left common carotid arteries (anterior view): 1. Innominate artery. 2. Left common carotid artery. 3. Ascending aorta. 4. Pulmonary artery. 5. Trachea. B: The pulmonary artery continues as a descending thoracic aorta via a widely dilated ductus arteriosus (upper view): 1 . Innominate artery. 2. Left common carotid artery. 3. Pulmonary artery. 4 . Right pulmonary artery. 5 . Left pulmonary artery. 6 . Dilated ductus arteriosus. 7 . Descending thoracic aorta. 8. Left subclavian ostium.

infection. Studies have provided evidence that placental inflammation may be associated with fetal growth abnormalities culminating in stillbirth. ${ }^{10}$ Infarcts are the most common lesions seen in the placenta. Large parenchymal infarctions identified in any location of the placenta cause placental abnormalities. ${ }^{11}$ In the 


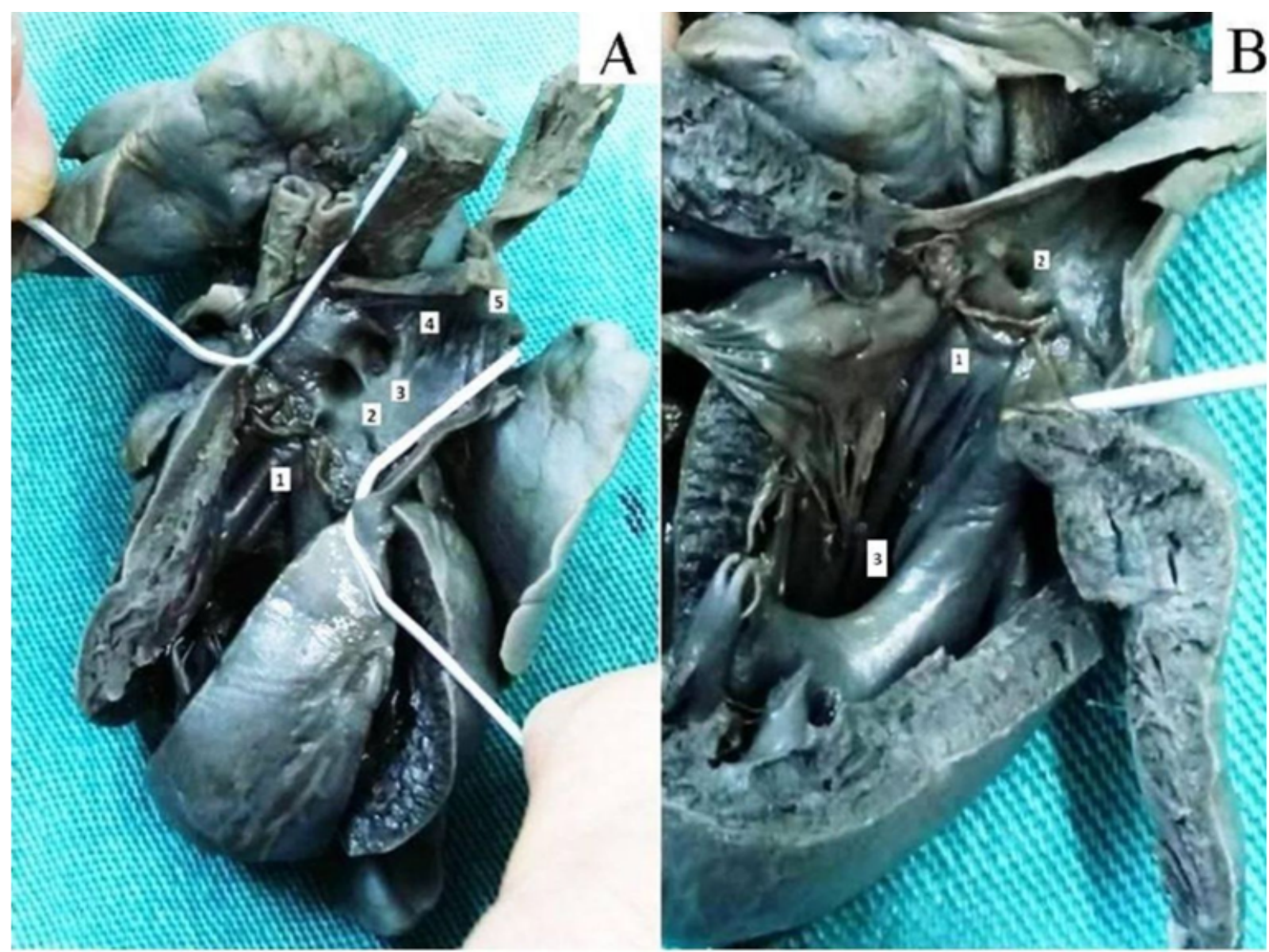

Figure 2 - A: Pulmonary valve, ostium of the left pulmonary artery (below) and ostium of the right pulmonary artery (above). 1. Pulmonary valve. 2. Ostium of the left pulmonary artery. 3. Ostium of the right pulmonary artery. 4. Ductus arteriosus. 5 . Descending thoracic aorta. B: Aortic valve and single coronary artery ostium. 1. Aortic valve. 2. Single coronary artery ostium. 3. Complete atrioventricular septal defect with common atrioventricular valve

reported case, we hypothesized that the combination of chorioamnionitis and placental infarction with reduced oxygenation, pertinent to the IAA, may have caused anemia, hidrops, and intrauterus heart failure that culminated in fetal death. Failure to perform the fetal karyotype analysis was a limitation of the reported case.

An important echocardiographic finding in the prenatal diagnosis of IAA is the size discrepancy between the large arteries, with the aortic artery being much smaller in diameter than the main pulmonary artery. In all these cases, it is important to check the continuity of the aortic arch. ${ }^{6}$ This difference in diameter between the great vessels was readily observed in the autopsy of the case described, but fetal echocardiography was not performed.
This case is particularly interesting because it emphasizes the importance of a detailed diagnostic investigation in suspicion of fetal hydrops in gestational ultrasound. Although cases of IAA are rare, this entity should be considered as a possible cause of fetal hydrops.

\section{Author contributions}

Conception and design of the research: Ponce C. Acquisition of data: Ponce C. Analysis and interpretation of the data: Ponce C. Statistical analysis: Ponce C. Obtaining financing: Ponce $\mathrm{C}$. Writing of the manuscript: Ponce C. Critical revision of the manuscript for intellectual content: Ponce C. Supervision / as the major investigador: Ponce $\mathrm{C}$. 


\section{Potential Conflict of Interest}

No potential conflict of interest relevant to this article was reported.

\section{Sources of Funding}

There were no external funding sources for this study.

\section{Study Association}

This study is not associated with any thesis or dissertation work.

\section{References}

1. Everts-Suarez EA, Carson CP. The triad of congenital absence of aortic arch (isthmus aortae), patent ductus arteriosus and interventricular septal defect; a trilogy. Ann Surg.1959;150(1):153-9.

2. Kumanan T, Guruparan M, Gnanakanthan K, Ratnayake UK. Interrupted aortic arch in an adult. Ceylon Med J. 2016;61(3):135-6.

3. Kattea MO, Smettei OA, Kattea A, Abazid RM. Interrupted aortic arch with isolated persistent left superior vena cava in patient with Turners syndrome. Avicenna J Med. 2016;6(4):117-9.

4. Reardon MJ, Hallman GL, Cooley DA. Interrupted aortic arch: brief review and summary of an eighteen-year experience. Tex Heart Inst J.1984;11(3):250-9.

5. Gáspár I. Two of the rarer congenital anomalies of the heart. Am J Pathol. 1929;5(3):285-294.3

6. Volpe P, Tuo G, De Robertis V, Campobasso G, Marasini M, Tempesta A, et al. Fetal interrupted aortic arch: 2D-4D echocardiography, associations and outcome. Ultrasound Obstet Gynecol. 2010;35(3):302-9.

\section{Ethics approval and consent to participate}

This study was approved by the Ethics Committee of the Universidade Metropolitana de Santos under the protocol number 2.965.717. All the procedures in this study were in accordance with the 1975 Helsinki Declaration, updated in 2013. Informed consent was obtained from all participants included in the study.

7. Bove KE. Practice guidelines for autopsy pathology: the perinatal and pediatric autopsy. Autopsy Committee of the College of American Pathologists. Arch Pathol Lab Med.1997;121(4):368-76.

8. Rastelli G, Kirklin JW, Titus JL. Anatomic observations on complete form of persistent common atrioventricular canal with special reference to atrioventricular valves. Mayo Clin Proc. 1966;41(5):296-308.

9. Celoria GC, Patton RB. Congenital absence of the aortic arch. Am Heart J. 1959 Sep;58:407-13.

10. Bukowski R, Hansen NI, Pinar H, Willinger M, Reddy UM, Parker CB, et al. Altered fetal growth, placental abnormalities, and stillbirth. PLoS One. 2017;12(8):e0182874.

11. Pinar H, Koch MA, Hawkins H, Heim-Hall J, Shehata B, Thorsten VR, et al. The Stillbirth Collaborative Research Network (SCRN) placental and umbilical cord examination protocol. Am J Perinatol. 2011;28(10):781-92. 